\title{
The Banks-Zaks expansion in perturbative QCD: an update
}

\author{
P. M. Stevenson \\ T.W. Bonner Laboratory, Department of Physics and Astronomy, \\ Rice University, Houston, TX 77251, USA
}

\begin{abstract}
:
The recent QCD calculations of the five-loop $\beta$-function and of $R_{e^{+} e^{-}}$to $O\left(\alpha_{s}^{4}\right)$ provide one more term in the Banks-Zaks expansion in $\left(16 \frac{1}{2}-n_{f}\right)$. There is no longer any hope that the expansion could extend, even crudely, to low $n_{f}$. Above $n_{f} \sim 9$, however, the results appear to be reasonably consistent from order to order.
\end{abstract}


This Letter is to update earlier work [1, 2, taking into account the new results of Baikov, Chetyrkin, and Kühn for the fifth-order $\beta$ function [3] and for $R_{e^{+} e^{-}}$at fourth order [4]. Contrary to our original hopes, the Banks-Zaks (BZ) expansion [5]-[7] appears to break down around $n_{f} \sim 9$ or sooner, and does not extrapolate, even crudely, to low $n_{f}$.

We write the $\beta$ function in the form:

$$
\beta(a) \equiv \mu \frac{d a}{d \mu}=-b a^{2}\left(1+c a+c_{2} a^{2}+c_{3} a^{3}+c_{4} a^{4}+\ldots\right)
$$

where $a \equiv \alpha_{s} / \pi$. The coefficients, in the $\overline{\mathrm{MS}}$ scheme, are [8]-[11], [3]:

$$
\begin{aligned}
2 b= & 11-\frac{2}{3} n_{f}, \\
8 b c= & 102-\frac{38}{3} n_{f}, \\
32 b c_{2}= & \frac{2857}{2}-\frac{5033}{18} n_{f}+\frac{325}{54} n_{f}^{2}, \\
128 b c_{3}= & \left.\frac{149753}{6}+3564 \zeta_{3}\right)-\left(\frac{1078361}{162}+\frac{6508}{27} \zeta_{3}\right) n_{f} \\
& +\left(\frac{50065}{162}+\frac{6472}{81} \zeta_{3}\right) n_{f}^{2}+\frac{1093}{729} n_{f}^{3}, \\
512 b c_{4}= & \left.\frac{8157455}{16}+\frac{621885}{2} \zeta_{3}-\frac{88209}{2} \zeta_{4}-288090 \zeta_{5}\right) \\
& +\left(-\frac{336460813}{1944}-\frac{4811164}{81} \zeta_{3}+\frac{33935}{6} \zeta_{4}+\frac{1358995}{27} \zeta_{5}\right) n_{f} \\
& +\left(\frac{25960913}{1944}+\frac{698531}{81} \zeta_{3}-\frac{10526}{9} \zeta_{4}-\frac{381760}{81} \zeta_{5}\right) n_{f}^{2} \\
& +\left(-\frac{630559}{5832}-\frac{48722}{243} \zeta_{3}+\frac{1618}{27} \zeta_{4}+\frac{460}{9} \zeta_{5}\right) n_{f}^{3}+\left(\frac{1205}{2916}-\frac{152}{81} \zeta_{3}\right) n_{f}^{4} .
\end{aligned}
$$

Here $\zeta_{s}$ is the Riemann zeta-function and $n_{f}$ is the number of massless quark flavours.

For $n_{f}$ just below $16 \frac{1}{2}$, the $\beta$ function has a zero at $a^{*} \sim-\frac{1}{c}$, and $a^{*}$ is asymptotically proportional to $\left(16 \frac{1}{2}-n_{f}\right)$. Its limiting form,

$$
a_{0} \equiv \frac{8}{321}\left(16 \frac{1}{2}-n_{f}\right)
$$

is the natural expansion parameter for the BZ expansion [1]. To proceed, one re-writes all perturbative coefficients, eliminating $n_{f}$ in favour of $a_{0}$. The first two $\beta$-function coefficients, which are renormalization-scheme (RS) invariant, become:

$$
b=\frac{107}{8} a_{0}, \quad c=-\frac{1}{a_{0}}+\frac{19}{4} .
$$


Within the class of so-called 'regular' schemes [7, 1], which includes $\overline{\mathrm{MS}}$, perturbative coefficients have a polynomial dependence on $n_{f}$, and we may write

$$
c_{i}=\frac{1}{a_{0}}\left(c_{i,-1}+c_{i, 0} a_{0}+c_{i, 1} a_{0}^{2}+\ldots\right) .
$$

The coefficients, in $\overline{\mathrm{MS}}$, are collected in the table below.

\begin{tabular}{rlrr|}
\hline$c_{1,0}$ & $=\frac{19}{4}$ & $=$ & 4.75 \\
\hline$c_{2,-1}$ & $=-\left(\frac{8}{107}\right)\left(\frac{37117}{768}\right)$ & $=$ & -3.61 \\
$c_{2,0}$ & $=\frac{243}{32}$ & $=$ & 7.59 \\
$c_{2,1}$ & $=\left(\frac{107}{8}\right)\left(\frac{325}{192}\right)$ & $=$ & 22.6 \\
\hline$c_{3,-1}=\left(\frac{8}{107}\right)\left(\frac{53981}{1152}+\frac{5335}{32} \zeta_{3}\right)$ & $=$ & -179 \\
$c_{3,0}$ & $=-\frac{1544327}{13824}-\frac{16171}{288} \zeta_{3}$ & & 451 \\
$c_{3,1}$ & $=\left(\frac{107}{8}\right)\left(\frac{2587}{96}+\frac{809}{144} \zeta_{3}\right)$ & $=$ & -56.6 \\
$c_{3,2}$ & $=-\left(\frac{107}{8}\right)^{2}\left(\frac{1093}{3456}\right)$ & $=$ & 156.7 \\
$c_{4,-1}$ & $=\left(\frac{8}{107}\right)\left(\frac{1081830511}{663552}+\frac{17251949}{13824} \zeta_{3}-\frac{191675}{192} \zeta_{5}\right)$ & $=$ & -1005.3 \\
$c_{4,0}$ & $=-\frac{1452057293}{1327104}-\frac{48015}{512} \zeta_{4}-\frac{4489165}{27648} \zeta_{3}+\frac{856625}{2304} \zeta_{5}$ & & 731.1 \\
$c_{4,1}$ & $=\left(\frac{107}{8}\right)\left(\frac{33737869}{22184}+\frac{16171}{512} \zeta_{4}-\frac{176837}{2304} \zeta_{3}-\frac{88415}{2304} \zeta_{5}\right)$ & $=$ & 3329.0 \\
$c_{4,2}$ & $=\left(\frac{107}{8}\right)^{2}\left(\frac{471499}{110592}-\frac{809}{256} \zeta_{4}+\frac{39409}{2304} \zeta_{3}-\frac{345}{128} \zeta_{5}\right)$ & $=$ & -697.4 \\
$c_{4,3}$ & $=\left(\frac{107}{8}\right)^{3}\left(\frac{1205}{18432}-\frac{19}{64} \zeta_{3}\right)$ & $=$ & \\
\hline
\end{tabular}

Table 1. $\beta$-function coefficients in the $\overline{\mathrm{MS}}$ scheme.

The BZ expansion can be applied to any perturbatively calculable physical quantity of the form:

$$
\mathcal{R}=a\left(1+r_{1} a+r_{2} a^{2}+r_{3} a^{3}+\ldots\right) .
$$

For 'primary' quantities calculated in a 'regular' scheme the coefficients $r_{i}$ are polynomials in $n_{f}$, and hence in $a_{0}$ :

$$
r_{i}=r_{i, 0}+r_{i, 1} a_{0}+r_{i, 2} a_{0}^{2}+\ldots
$$

Note that a term $r_{i, j} a_{0}^{p}$ or $c_{i, j} a_{0}^{p}$ can be assigned a degree $i+j-p$, and all terms in any formula must have matching degree. [We mention that the same decomposition of coefficients is needed in the "large- $b$ " approximation [12, 13], which employs the opposite limit $(b \rightarrow \infty)$, rather than $b=\frac{107}{8} a_{0} \rightarrow 0$ as here.]

The prototypical example is the $e^{+} e^{-}$ratio at a $\mathrm{cm}$ energy $Q$ :

$$
R_{e^{+} e^{-}}(Q) \equiv \frac{\sigma_{\text {tot }}\left(e^{+} e^{-} \rightarrow \text { hadrons }\right)}{\sigma\left(e^{+} e^{-} \rightarrow \mu^{+} \mu^{-}\right)},
$$


where, neglecting quark masses, $R_{e^{+} e^{-}}(Q)=3 \Sigma q_{i}^{2}\left(1+\mathcal{R}_{e^{+} e^{-}}\right)$, and $\mathcal{R}_{e^{+} e^{-}}$has the form of Eq. (6). [We will drop "singlet" terms proportional to $\left(\Sigma q_{i}\right)^{2} /\left(3 \Sigma q_{i}^{2}\right)$ whose $n_{f}$ dependence is ambiguous and depends on the electric charges assigned to the additional, fictitious quarks.] The coefficients, calculated in $\overline{\mathrm{MS}}$ with the renormalization scale $\mu$ equal to $Q$ [14, 15, 4], are collected in the table below.

\begin{tabular}{|rlr|}
\hline$r_{1,0}=\frac{1}{12}$ & $=$ & 0.0833 \\
$r_{1,1}=\left(\frac{107}{8}\right)\left(\frac{11}{4}-2 \zeta_{3}\right)$ & $=$ & 4.63 \\
\hline$r_{2,0}=-\frac{12521}{288}+13 \zeta_{3}$ & $=$ & -27.85 \\
$r_{2,1}=\left(\frac{107}{8}\right)\left(\frac{401}{24}-\frac{53}{3} \zeta_{3}+\frac{25}{3} \zeta_{5}\right)$ & $=$ & 55.0 \\
$r_{2,2}=\left(\frac{107}{8}\right)^{2}\left(\frac{151}{18}-\frac{19}{3} \zeta_{3}-\frac{1}{2} \zeta_{2}\right)$ & $=$ & -8.34 \\
\hline$r_{3,0}=-\frac{3963761}{20736}+\frac{677833}{3456} \zeta_{3}-\frac{275}{24} \zeta_{5}$ & $=$ & 32.73 \\
$r_{3,1}=\left(\frac{107}{8}\right)\left(-\frac{38969}{128}+\frac{535}{32} \zeta_{2}+\frac{6907}{96} \zeta_{3}+\frac{165}{2} \zeta_{3}^{2}+\frac{9595}{144} \zeta_{5}-\frac{665}{24} \zeta_{7}\right)$ & $=$ & -402.6 \\
$r_{3,2}=\left(\frac{107}{8}\right)^{2}\left(\frac{236089}{1728}-\frac{97}{16} \zeta_{2}-\frac{13859}{96} \zeta_{3}+\frac{15}{2} \zeta_{3}^{2}+\frac{445}{12} \zeta_{5}\right)$ & $=$ & 430.9 \\
$r_{3,3}=\left(\frac{107}{8}\right)^{3}\left(\frac{6131}{216}-\frac{33}{8} \zeta_{2}-\frac{203}{12} \zeta_{3}+3 \zeta_{2} \zeta_{3}-\frac{15}{2} \zeta_{5}\right)$ & $=$ & -1390.0 \\
\hline
\end{tabular}

Table 2. Coefficients in $\mathcal{R}_{e^{+} e^{-}}$in the $\overline{\mathrm{MS}}(\mu=Q)$ scheme.

The fixed-point condition $\beta\left(a^{*}\right)=0$ always has a solution as a power series in $a_{0}$ :

$$
a^{*}=a_{0}\left(1+v_{1} a_{0}+v_{2} a_{0}^{2}+v_{3} a_{0}^{3}+\ldots\right) .
$$

A straightforward calculation yields:

$$
\begin{aligned}
v_{1}= & c_{1,0}+c_{2,-1}, \\
v_{2}= & \left(c_{1,0}+2 c_{2,-1}\right)\left(c_{1,0}+c_{2,-1}\right)+c_{2,0}+c_{3,-1}, \\
v_{3}= & c_{1,0}^{3}+6 c_{1,0}^{2} c_{2,-1}+c_{1,0}\left(3 c_{2,0}+4 c_{3,-1}+10 c_{2,-1}^{2}\right) \\
& +c_{2,-1}\left(4 c_{2,0}+5 c_{3,-1}\right)+5 c_{2,-1}^{3}+c_{2,1}+c_{3,0}+c_{4,-1} .
\end{aligned}
$$

Numerically, $v_{1}=1.1366, v_{2}=23.27, v_{3}=18.10$, in the $\overline{\mathrm{MS}}$ scheme. Since $a^{*}$ is RS dependent, the good or bad convergence of this series need not concern us.

A physical quantity $\mathcal{R}$ also has an infrared limit, $\mathcal{R}^{*}$, given by a power series in $a_{0}$. Substituting $a=a^{*}$ from Eq. (9) into the perturbative expansion of $\mathcal{R}$ and re-expanding in powers of $a_{0}$ yields

$$
\mathcal{R}^{*}=a_{0}\left(1+w_{1} a_{0}+w_{2} a_{0}^{2}+w_{3} a_{0}^{3}+\ldots\right),
$$


where

$$
\begin{aligned}
& w_{1}=v_{1}+r_{1,0} \\
& w_{2}=v_{2}+2 r_{1,0} v_{1}+r_{2,0}+r_{1,1}, \\
& w_{3}=v_{3}+\left(2 v_{2}+v_{1}^{2}\right) r_{1,0}+v_{1}\left(2 r_{1,1}+3 r_{2,0}\right)+r_{2,1}+r_{3,0} .
\end{aligned}
$$

These coefficients are RS independent. For the $e^{+} e^{-}$case they are

$$
\begin{aligned}
& w_{1}=\frac{4177}{2^{5}(107)}, \\
& w_{2}=\frac{31250575}{2^{9} 3(107)^{2}}-\frac{275}{2(107)} \zeta_{3}, \\
& w_{3}=\frac{2177185161509}{2^{15} 3^{2}(107)^{3}}-\frac{4232749}{2^{6}(107)^{2}} \zeta_{3}+\frac{65125}{2^{3} 3(107)} \zeta_{5} .
\end{aligned}
$$

Numerically we find

$$
\mathcal{R}_{e^{+} e^{-}}^{*}=a_{0}\left(1+1.22 a_{0}+0.23 a_{0}^{2}+25.38 a_{0}^{3}+\ldots\right) .
$$

While the first three terms raise hopes for a well-behaved series, those hopes are dashed by the last term. See Fig. 1.

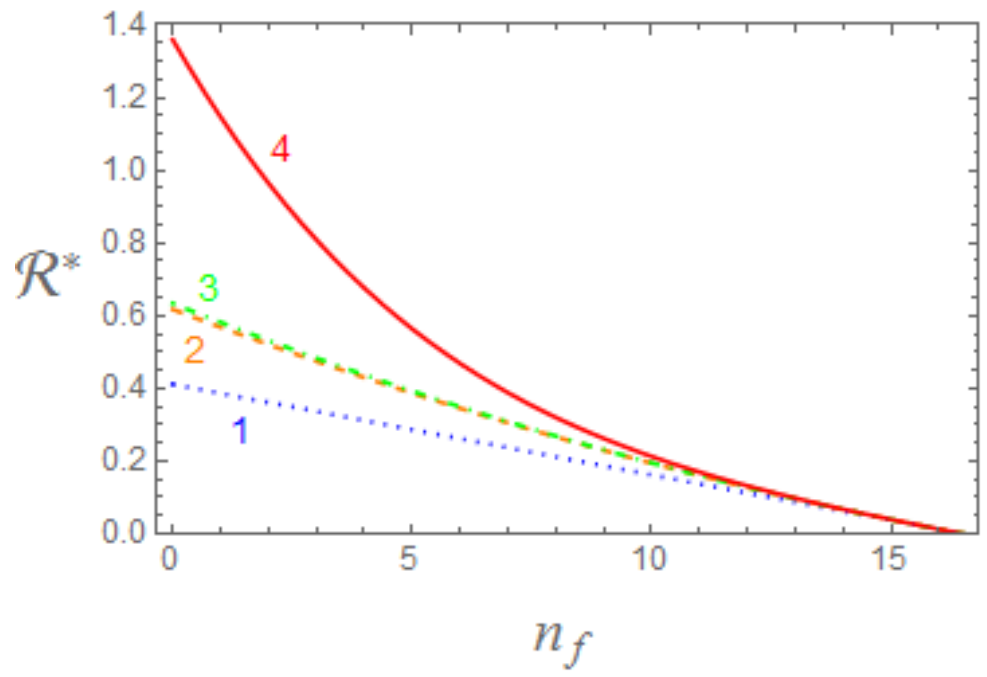

Fig. 1. $\mathcal{R}^{*}$ as a function of $n_{f}$ in the BZ expansion. The curves for $1^{\text {st }}$ to $4^{\text {th }}$ order are shown dotted, dashed, dot-dashed, and solid.

A formulation of the BZ expansion for quantities at a general $Q$ was derived in Ref. [1]. First, we write the integrated $\beta$-function equation in the form

$$
b \ln \left(\frac{\mu}{\tilde{\Lambda}}\right)=\frac{1}{a}+c \ln (|c| a)+\int_{0}^{a} d x\left(\frac{b}{\beta(x)}+\frac{1}{x^{2}}-\frac{c}{x}\right) .
$$


This form, more convenient for $c$ negative, is completely equivalent to our previous definition of the $\tilde{\Lambda}$ parameter [16, 2]. We use a tilde to distinguish $\tilde{\Lambda}$ from the conventional definition of the $\Lambda$ parameter [17]. The relation is $\ln (\Lambda / \tilde{\Lambda})=(c / b) \ln (2|c| / b)$. The two definitions are not dissimilar for small $n_{f}$, but they become infinitely different as $n_{f} \rightarrow 16 \frac{1}{2}$. In the BZ-expansion context the use of $\tilde{\Lambda}$ is much more convenient.

As explained in Ref. [1, it is convenient to put the $\beta$ function into the form

$$
\frac{b}{\beta(x)}=-\frac{1}{x^{2}}+\frac{c}{x}-\frac{b}{\gamma^{*}\left(a^{*}-x\right)}+H(x) .
$$

where $\gamma^{*}$ is the slope of the $\beta$ function at the fixed point:

$$
\left.\gamma^{*} \equiv \frac{d \beta(x)}{d x}\right|_{x=a^{*}}=-b a^{*}\left(1+2 c a^{*}+3 c_{2} a^{* 2}+4 c_{3} a^{* 3}+\ldots\right) .
$$

As discussed below, $\gamma^{*}$ can be obtained as a series in $a_{0}$. The remainder function $H(x)$ can be expanded as a power series, $H_{0}+H_{1} x+\ldots$, whose coefficients are of order $a_{0}$.

One now inserts (16) into 15 and performs the integration. One can then eliminate $a$ and $a^{*}$ in favour of $\mathcal{R}$ and $\mathcal{R}^{*}$. In fact, since the result must be RS invariant, one can without loss of generality — short-cut this step by utilizing the "effective-charge" RS [18] in which $a \equiv \mathcal{R}$. In $n^{t h}$ order of the BZ expansion this leads to the formula [1]:

$$
\boldsymbol{\rho}_{1}(Q)=\frac{1}{\mathcal{R}}+c \ln (|c| \mathcal{R})+\frac{b}{\gamma^{*(n)}} \ln \left(1-\frac{\mathcal{R}}{\mathcal{R}^{*(n)}}\right)+\sum_{i=0}^{n-4} \frac{H_{i}^{(\mathrm{EC})} \mathcal{R}^{i+1}}{i+1} .
$$

On the left-hand side, $\boldsymbol{\rho}_{1}(Q)$ is the RS invariant

$$
\rho_{1}(Q) \equiv b \ln \left(\frac{\mu}{\tilde{\Lambda}}\right)-r_{1} \equiv b \ln \left(\frac{Q}{\tilde{\Lambda}_{\mathcal{R}}}\right)
$$

where $\tilde{\Lambda}_{\mathcal{R}}$ is a characteristic scale specific to the particular physical quantity $\mathcal{R}$. It is related to the $\tilde{\Lambda}$ parameter of some reference scheme (eg. $\overline{\mathrm{MS}}$ ) by an exactly calculable factor $\exp \left(r_{1}(\mu=Q) / b\right)$ involving the $r_{1}$ coefficient in that scheme, evaluated at $\mu=Q$. On the right-hand side the terms involving the $H_{i}^{(\mathrm{EC})}$ coefficients of the effective-charge scheme are only relevant in fourth order and beyond. Thus, for the first three orders the equation takes the same form, just with the parameters $\gamma^{*}$ and $\mathcal{R}^{*}$ approximated to the appropriate order. At $4^{\text {th }}$ order there is an extra term $H_{0}^{(\mathrm{EC})} \mathcal{R}$, with $H_{0}^{(\mathrm{EC})}=H_{0,1}^{(\mathrm{EC})} a_{0}+O\left(a_{0}^{2}\right)$, where

$$
\begin{aligned}
H_{0,1}^{(\mathrm{EC})}= & \rho_{4,-1}+2 \rho_{2,-1} \rho_{3,-1}+\rho_{2,-1}^{3}, \\
= & c_{4,-1}+c_{2,-1}\left(2 c_{3,-1}+r_{1,0}^{2}-r_{2,0}\right)+c_{2,-1}^{3} \\
& -c_{2,-1}^{2} r_{1,0}-c_{3,-1} r_{1,0}-r_{1,0}^{3}+2 r_{1,0} r_{2,0}-r_{3,0}, \\
= & \frac{243227350299721}{2^{15} 3^{4}(107)^{3}}-\frac{5729638277}{2^{7} 3^{3}(107)^{2}} \zeta_{3}-\frac{81125}{2^{2} 3(107)} \zeta_{5} \simeq-164.8
\end{aligned}
$$


(in the first line, the $\rho_{i, j}$ are the $\beta$-function coefficients of the EC scheme).

The BZ expansion for $\gamma^{*}$ is obtained straightforwardly by substituting the expansion of $a^{*}$ (Eqs. (9) and (10) into (17). This gives:

$$
\gamma^{*}=b a_{0}\left(1+g_{1} a_{0}+g_{2} a_{0}^{2}+g_{3} a_{0}^{3}+\ldots\right),
$$

with

$$
\begin{aligned}
g_{1}= & c_{1,0}, \\
g_{2}= & c_{1,0}{ }^{2}-c_{2,-1}{ }^{2}-c_{3,-1}, \\
g_{3}= & c_{1,0}{ }^{3}-4 c_{2,-1}{ }^{3}-5 c_{1,0} c_{2,-1}{ }^{2}-4 c_{1,0} c_{3,-1} \\
& -2 c_{2,-1} c_{2,0}-6 c_{2,-1} c_{3,-1}-c_{3,0}-2 c_{4,-1} .
\end{aligned}
$$

It is noteworthy that certain terms of degree $n$ are absent in $g_{n}: g_{1}$ does not contain $c_{2,-1}$; $g_{2}$ does not contain $c_{2,0}$ or $c_{2,-1} c_{1,0}$; and $g_{3}$ does not contain $c_{2,1}$ or $c_{2,0} c_{1,0}$ or $c_{2,-1} c_{1,0}^{2}$.

The values of these invariants are

$$
\begin{aligned}
& g_{1}=\frac{19}{4}, \\
& g_{2}=\frac{633325687}{2^{10} 3^{2}(107)^{2}}-\frac{5335}{2^{2}(107)} \zeta_{3}, \\
& g_{3}=-\frac{66670528901419}{2^{13} 3^{4}(107)^{3}}-\frac{1920043907}{2^{6} 3^{3}(107)^{2}} \zeta_{3}+\frac{191675}{2^{2} 3(107)} \zeta_{5} .
\end{aligned}
$$

Numerically the $\gamma^{*}$ series is:

$$
\gamma^{*}=b a_{0}\left(1+4.75 a_{0}-8.98 a_{0}^{2}-43.89 a_{0}^{3}+\ldots\right)
$$

The results, at different orders, are shown in Fig. 2.

Note that $\gamma^{*}$ is the 'critical exponent' in the relation $\mathcal{R}^{*}-\mathcal{R} \propto Q^{\gamma^{*}}$ that describes how $\mathcal{R}$ approaches $\mathcal{R}^{*}$ as $Q \rightarrow 0$. ( $\gamma^{*}$ is the infrared limit of an RS-invariant 'effective exponent' $\gamma(Q) \equiv 1+Q \frac{d^{2} \mathcal{R}}{d Q^{2}} / \frac{d \mathcal{R}}{d Q}=\frac{d \beta}{d a}+\beta(a) \frac{d^{2} \mathcal{R}}{d a^{2}} / \frac{d \mathcal{R}}{d a}[19]$.) As pointed out by Grunberg [7], the $g_{n}$ coefficients are RS invariants and are universal, in the sense that they are not specific to a particular physical quantity $\mathcal{R}$.

Numerically inverting Eq. (18) provides $\mathcal{R}$ as a function of $Q$. In the BZ region, $n_{f} \gtrsim 9$, the resulting $\mathcal{R}(Q)$ has the general form sketched in Fig. 3. At large $Q$ the result naturally agrees with ordinary perturbation theory to the corresponding order. For $Q \sim \tilde{\Lambda}_{\mathcal{R}}$ there is a large "sloping plateau" region, and at ultra-low energies there is a "spike" reaching up to $\mathcal{R}^{*(n)}$. 


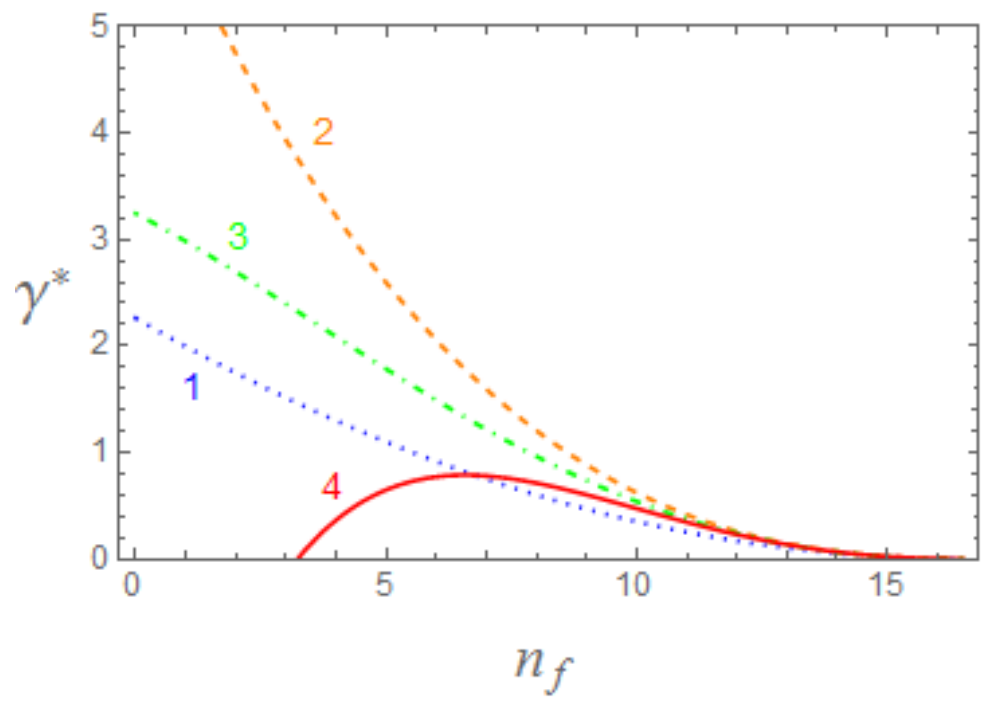

Fig. 2. $\gamma^{*}$ as a function of $n_{f}$ in the BZ expansion. The curves for $1^{\text {st }}$ to $4^{\text {th }}$ order are shown dotted, dashed, dot-dashed, and solid.

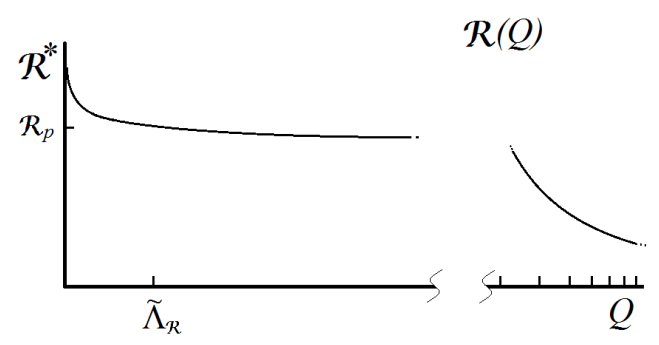

Fig. 3. Typical appearance of $\mathcal{R}$ as a function of $Q$ in the BZ region $\left(n_{f} \gtrsim 9\right)$ showing the "spike" at very low energies, the "sloping plateau" region, and the slow approach to asymptotic freedom at very high energies (this region is shown on a $\log$ scale). The plateau value $\mathcal{R}_{p}$ is generally about 0.8 times $\mathcal{R}^{*}$ but depends on $n_{f}$ and the BZ-expansion order.

We conclude by showing, in Fig. 4 , a comparison of the $4^{\text {th }}$ order BZ expansion with the $\mathcal{R}_{e^{+} e^{-}}^{*}$ results of Ref. [20] in optimized perturbation theory (OPT) [16] and in the EC scheme [18] to order $\alpha_{s}^{4}$. Contrary to the conjecture of Refs. [1, 2], it now appears that the "freezing" behaviour of $\mathcal{R}_{e^{+} e^{-}}^{*}$ at low $n_{f}$ [21, 22] is not an extrapolation from the BZ region, but a distinct phenomenon. 1] At low $n_{f}$ one finds that $\gamma^{*}$ is around 2 or 3 , so that $\mathcal{R}$ "freezes," becoming nearly constant in the infrared region, while it falls rapidly around

${ }^{1}$ At low $n_{f}$ it appears that different physical quantities may have rather different infrared behaviours 23], unlike the BZ region where there is a high degree of universality. 


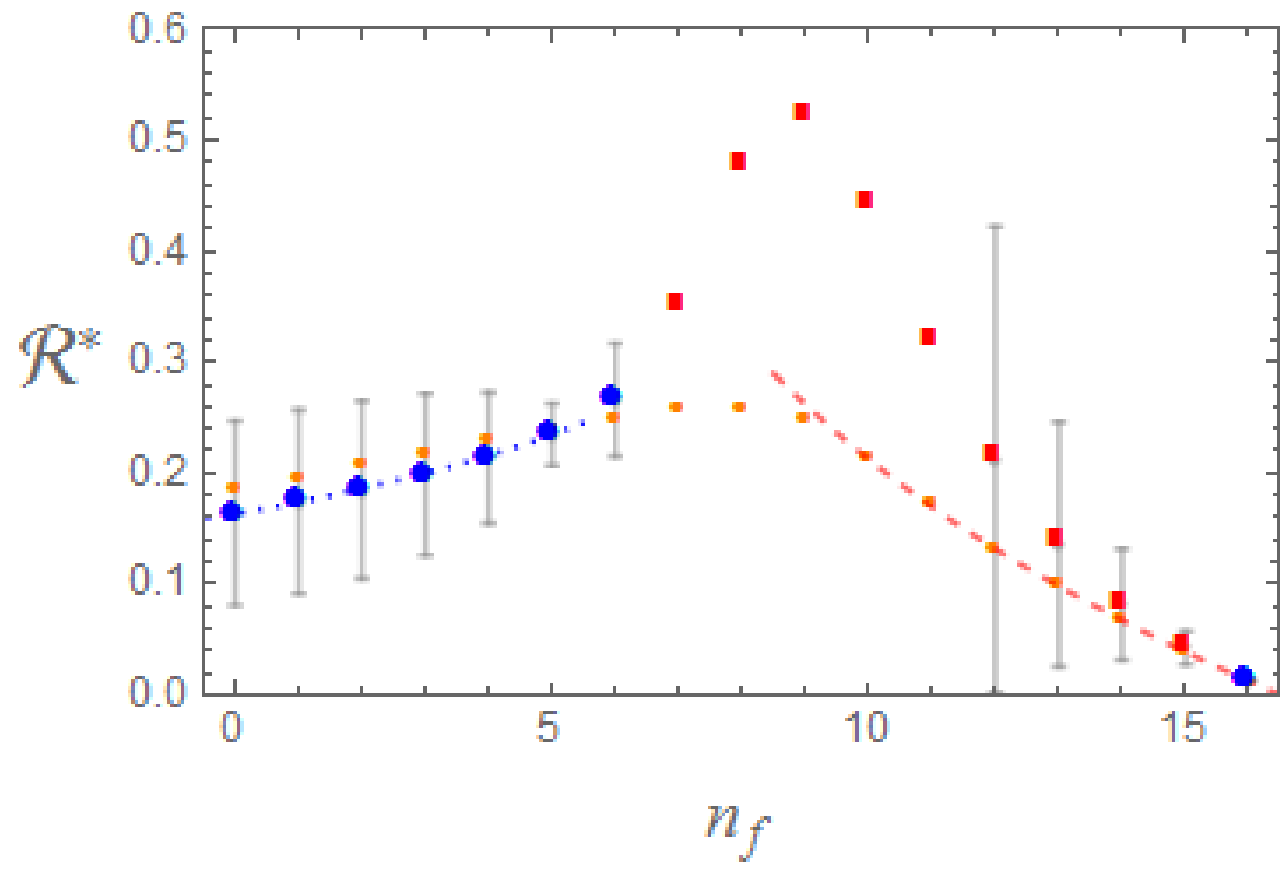

Fig. 4. $\quad \mathcal{R}^{*}$ as a function of $n_{f}$ in the $4^{\text {th }}$ order BZ expansion (dashed curve) compared with OPT (large points) and EC (small points) results from Ref. [20]. The OPT points are shown as blue circles when they arise from a fixed point and as red squares when they arise from an "unfixed point." Error bars indicate the estimated uncertainty in the OPT results. (They are not shown for $n_{f}=$ $7, \ldots, 11$, where they would extend well beyond the bounds of the plot.) The dotted blue curve represents $\mathcal{R}^{*}=0.9 / b$, a purely speculative guess at the large- $b$ form.

$Q \simeq \tilde{\Lambda}_{\mathcal{R}}$. In the BZ region, by contrast, $\gamma^{*}$ is small $(\lesssim 1)$, resulting in the infrared "spike" of Fig. 3 and the sloping plateau around $Q \simeq \tilde{\Lambda}_{\mathcal{R}}$.

The OPT and EC results in Fig. 4 agree remarkably well at both low and high $n_{f}$. In the intermediate region $7 \lesssim n_{f} \lesssim 13$ they actually differ only at the very lowest energies, because OPT indicates a much more dramatic "spike" in the infrared, of very uncertain size - it could well be even bigger than predicted. This is because the infrared limit in OPT here does not result from a fixed point but from an "unfixed point" and a "pinch mechanism" that leads to $\left(\mathcal{R}^{\star}-\mathcal{R}\right) \propto 1 /|\ln Q|^{2}$, corresponding to $\gamma^{*}=0$. For details, see Ref. [20].

Acknowledgment: This note is partly based on unpublished work done in collaboration with Scott Caveny. 


\section{References}

[1] P. M. Stevenson, Phys. Lett. B 331, 187 (1994).

[2] S. A. Caveny and P. M. Stevenson, hep-ph/9705319 (unpublished).

[3] P. A. Baikov, K. G. Chetyrkin, and J. H. Kühn, arXiv 1606.08659 [hep-ph].

[4] P. A. Baikov, K. G. Chetyrkin, J. H. Kühn, and J. Rittinger, arXiv:1206:1288 (2012);

P. A. Baikov, K. G. Chetyrkin, and J. H. Kühn, Phys. Rev. Lett. 101, 012002 (2008).

[5] T. Banks and A. Zaks, Nucl. Phys. B 196, 189 (1982).

[6] A. R. White, Phys. Rev. D 29, 1435 (1984); in Hadronic Matter in Collision, edited by J. Rafelski (World Scientific, 1989); Int. J. Mod. Phys. A 8, 4755 (1993).

[7] G. Grunberg, Phys. Rev. D 46, 2228 (1992).

[8] H. D. Politzer, Phys. Rev. Lett. 30, 1346 (1973); D. J. Gross and F. Wilczek, ibid. 30, 1343 (1973); G. 't Hooft, report at the Marseille Conference Yang-Mills Fields, 1972.

[9] D. R. T. Jones, Nucl. Phys. B 75, 531 (1974); W. Caswell, Phys. Rev. Lett. 33, 244 (1974); E. S. Egorian and O. V. Tarasov, Theor. Mat. Fiz. 41, 26 (1979).

[10] O. V. Tarasov, A. A. Vladimirov, and A. Yu. Zharkov, Phys. Lett. B 93, 429 (1980); S. A. Larin and J. A. M. Vermaseren, Phys. Lett. B 303, 334 (1993).

[11] T. van Ritbergen, J. A. M. Vermaseren, and S. A. Larin, Phys. Lett. B 400, 379 (1997).

[12] M. Beneke, Nucl. Phys. B 405, 424 (1993); D. J. Broadhurst, Z. Phys. C 58, 339 (1993); K. Van Acoleyen and H. Verschelde, Phys. Rev. D 69125006 (2004).

[13] C. N. Lovett-Turner and C. J. Maxwell, Nucl. Phys. B 432, 147 (1994); ibid 452, 188 (1995); C. J. Maxwell and D. G. Tonge, ibid 481, 681 (1996); P. M. Brooks and C. J. Maxwell, Phys. Rev. D 74, 065012 (2006).

[14] K. G. Chetyrkin, A. L. Kataev, and F. V. Tkachov, Phys. Lett. B 85, 277 (1979); M. Dine and J. Sapirstein, Phys. Rev. Lett. 43, 668 (1979); W. Celmaster and R. J. Gonsalves, Phys. Rev. D 21, 3112 (1980).

[15] L. R. Surguladze and M. A. Samuel, Phys. Rev. Lett. 66, 560 (1991); ibid 2416 (E);

S. G. Gorishny, A. L. Kataev, and S. A. Larin, Phys. Lett. B 259, 144 (1991). 
[16] P. M. Stevenson, Phys. Rev. D23, 2916 (1981).

[17] W. A. Bardeen, A. J. Buras, D. W. Duke, and T. Muta, Phys. Rev. D 18, 3998 (1978).

[18] G. Grunberg, Phys. Rev. D 29, 2315 (1984); A. Dhar and V. Gupta, Phys. Rev. D 29, 2822 (1984).

[19] P. M. Stevenson, arXiv: 1606.06951 [hep-ph].

[20] P. M. Stevenson, Nucl. Phys. B 875, 63 (2013).

[21] J. Chýla, A. Kataev, and S. A. Larin, Phys. Lett. B 267, 269 (1991).

[22] A. C. Mattingly and P. M. Stevenson, Phys. Rev. Lett. 69, 1320 (1992); Phys. Rev. D 49, 437 (1994); P. M. Stevenson, Nucl. Phys. B 868, 38 (2013).

[23] E. Gardi and M. Karliner, Nucl. Phys. B 529, 383 (1998). 